PROCEEDINGS OF THE

AMERICAN MATHEMATICAL SOCIETY

Volume 125, Number 6, June 1997, Pages 1711-1716

S 0002-9939(97)03753-2

\title{
BEALS-CORDES-TYPE CHARACTERIZATIONS OF PSEUDODIFFERENTIAL OPERATORS
}

\author{
MICHAEL E. TAYLOR
}

(Communicated by Christopher D. Sogge)

\begin{abstract}
We show that, if $U$ is the representation of $S O_{e}(n+1,1)$ on $L^{2}\left(S^{n}\right)$ given by (2.11), and $P$ is a bounded operator on $L^{2}\left(S^{n}\right)$, then $P$ belongs to $O P S_{1,0}^{0}\left(S^{n}\right)$ if and only if$$
P(g)=U(g) P U(g)^{-1}
$$

is a $C^{\infty}$ function on $S O_{e}(n+1,1)$ with values in the Banach space $\mathcal{L}\left(L^{2}\left(S^{n}\right)\right)$.
\end{abstract}

\section{INTRODUCTION}

Let $M$ be a compact $C^{\infty}$ manifold. Denote by $O P S_{1,0}^{m}(M)$ the space of pseudodifferential operators on $M$, whose symbols in local coordinates satisfy

$$
\left|D_{x}^{\beta} D_{\xi}^{\alpha} p(x, \xi)\right| \leq C_{\alpha \beta}\langle\xi\rangle^{m-|\alpha|},
$$

and denote by $O P S^{m}(M)$ the subspace of classical pseudodifferential operators, whose symbols have the asymptotic behavior

$$
p(x, \xi) \sim p_{m}(x, \xi)+p_{m-1}(x, \xi)+\cdots, \quad|\xi| \rightarrow \infty,
$$

with $p_{m-j}(x, \xi)$ homogeneous of degree $m-j$ in $\xi$.

It was demonstrated in [B], [C], and [D] that, if $P: L^{2}(M) \rightarrow L^{2}(M)$, then $P$ belongs to $O P S_{1,0}^{0}(M)$ if and only if, for arbitrary $A_{j} \in O P S^{1}(M)$, and any $N \in \mathbb{Z}^{+}$,

$$
\operatorname{ad} A_{N} \cdots \operatorname{ad} A_{1} \cdot P: L^{2}(M) \rightarrow L^{2}(M),
$$

where ad $A_{j} \cdot B=\left[A_{j}, B\right]$.

It is desirable to have some alternative characterizations, and we give one in $\S 2$ of this paper, which has as a special case the following (when $M=S^{n}$, the $n$-dimensional sphere):

Theorem. If $U$ is the representation of $S O_{e}(n+1,1)$ on $L^{2}\left(S^{n}\right)$ given by (2.11), and $P$ is a bounded operator on $L^{2}\left(S^{n}\right)$, then $P$ belongs to $O P S_{1,0}^{0}\left(S^{n}\right)$ if and only if

$$
P(g)=U(g) P U(g)^{-1}
$$

is a $C^{\infty}$ function on $S O_{e}(n+1,1)$ with values in the Banach space $\mathcal{L}\left(L^{2}\left(S^{n}\right)\right)$.

Received by the editors July 5, 1995 and, in revised form, December 6, 1995.

1991 Mathematics Subject Classification. Primary 35S05.

This work was partially supported by the National Science Foundation.

(C)1997 American Mathematical Society 
It is technically useful that this characterization involves smoothness on a finite dimensional Lie group. One implication is that the group of invertible operators in $O P S_{1,0}^{0}(M)$ forms a smooth tame Frechét Lie group (in the terminology of $[\mathrm{H}]$ ), by arguments such as used in $[\mathrm{P}]$. This in turn leads to a simplification and elucidation of some of the pseudodifferential operator estimates in [GY]. We plan to pursue this matter further, in another paper.

\section{Generalities; $\mathfrak{A}$-Smooth operators}

Let $\mathfrak{A}=\left\{A_{j}\right\}$ be a collection of operators in $O P S^{1}(M)$, for some compact smooth Riemannian manifold $M$. We will make the following two hypotheses on $\mathfrak{A}:$

$$
\begin{gathered}
\qquad A_{j}^{*}=A_{j}, \quad \bmod O P S^{0}(M) ; \\
\text { some finite sum } L=\sum A_{j}^{2} \text { is elliptic in } O P S^{2}(M) .
\end{gathered}
$$

An operator $P \in \mathcal{L}\left(L^{2}(M)\right)$ will be called $\mathfrak{A}$-smooth provided

$$
(\operatorname{ad} A)^{J} P \text { is bounded on } L^{2}(M),
$$

for each $(\operatorname{ad} A)^{J}=\operatorname{ad} A_{j_{l}} \cdots \operatorname{ad} A_{j_{1}}$, where ad $A \cdot P=[A, P]$. We use a common multi-index notation: $J=\left(j_{l}, \ldots, j_{1}\right)$, we also set $|J|=l$. A priori, the hypothesis $P: L^{2}(M) \rightarrow L^{2}(M)$ implies that $(\operatorname{ad} A)^{J} P: C^{\infty}(M) \rightarrow \mathcal{D}^{\prime}(M)$.

We mention a few very simple results, with almost trivial proofs.

Lemma 1.1. The set $\mathcal{S}_{\mathfrak{A}}$ of $\mathfrak{A}$-smooth operators is an algebra, containing $O P S_{1,0}^{0}(M)$.

Proof. That $\mathcal{S}_{\mathfrak{A}}$ is an algebra follows from the identity

$$
\left(\operatorname{ad} A_{j}\right)\left(P_{1} P_{2}\right)=P_{1}\left(\operatorname{ad} A_{j} \cdot P_{2}\right)+\left(\operatorname{ad} A_{j} \cdot P_{1}\right) P_{2} .
$$

Lemma 1.2. Let $\mathfrak{A}^{\prime}=\left\{A_{j}+B_{j}\right\}, B_{j} \in O P S^{0}(M)$. If $P$ is $\mathfrak{A}$-smooth, it is also $\mathfrak{A}^{\prime}$-smooth.

Lemma 1.3. If $P$ is $\mathfrak{A}$-smooth, so is $P^{*}$.

Proof. We have ad $A_{j} \cdot P^{*}=-\left(\operatorname{ad} A_{j}^{*} \cdot P\right)^{*}$, hence

$$
(\operatorname{ad} A)^{J} P^{*}=(-1)^{|J|}\left[\left(\operatorname{ad} A^{*}\right)^{J} P\right]^{*} .
$$

Lemma 1.4. If $P$ is $\mathfrak{A}$-smooth, so is each $(\operatorname{ad} A)^{J} P$.

Lemma 1.5. If $P$ is $\mathfrak{A}$-smooth, then $P$ and $(\operatorname{ad} A)^{J} P$ preserve each Sobolev space $H^{s}(M)$.

Proof. We have

$$
A_{k} P=P A_{k}+\text { ad } A_{k} \cdot P .
$$

Since the left side maps $L^{2}(M)$ to $H^{-1}(M)$, we have

$$
P A_{k}: L^{2}(M) \rightarrow H^{-1}(M) \text {. }
$$

By (1.2) it follows that $P: H^{-1}(M) \rightarrow H^{-1}(M)$. That $P: H^{1}(M) \rightarrow H^{1}(M)$ follows by duality, given Lemma 1.3. The rest follows by iteration and interpolation. 
We next note the approximate commutativity of $P$ with $L$. Indeed,

$$
[L, P]=\sum\left\{\left(\operatorname{ad} A_{j} \cdot P\right) A_{j}+A_{j}\left(\operatorname{ad} A_{j} \cdot P\right)\right\} .
$$

If $P$ is $\mathfrak{A}$-smooth, this maps $H^{s}(M)$ to $H^{s-1}(M)$. Hence

$$
\begin{aligned}
& L P L^{-1}-P=[L, P] L^{-1}, \\
& L^{-1} P L-P=L^{-1}[P, L],
\end{aligned}
$$

with each right side mapping $H^{s}(M)$ into $H^{s+1}(M)$.

If $P$ is $\mathfrak{A}$-smooth, it can often be established that $P$ is $\widetilde{\mathfrak{A}}$-smooth, for a much bigger collection of operators $\widetilde{\mathfrak{A}}$. We make some simple comments on this phenomenon here; more incisive results will be given in $\S 2$. The next result follows immediately from Jacobi's identity

$$
\text { ad } A_{1} \text { ad } A_{2}-\operatorname{ad} A_{2} \text { ad } A_{1}=\operatorname{ad}\left[A_{1}, A_{2}\right] .
$$

Lemma 1.6. Let $\mathfrak{L A}$ be the Lie algebra generated by $\mathfrak{A}$. If $P$ is $\mathfrak{A}$-smooth, then it is $\mathfrak{L} \mathfrak{A}$-smooth.

Generalizing the class $\mathcal{S}_{\mathfrak{A}}$ of $\mathfrak{A}$-smooth operators let us say, for an operator $P$ acting on $\mathcal{D}^{\prime}(M)$ and preserving $C^{\infty}(M)$, that

$$
P \in \mathcal{S}_{\mathfrak{A}}^{m} \Leftrightarrow(\operatorname{ad} A)^{J} P: H^{s}(M) \rightarrow H^{s-m}(M),
$$

for all commutators as in (1.3). Thus $\mathcal{S}_{\mathfrak{A}}^{0}$ is what is denoted $\mathcal{S}_{\mathfrak{A}}$ above. Parallel to Lemma 1.1, we clearly have

$$
P_{j} \in \mathcal{S}_{\mathfrak{A}}^{m_{j}} \Rightarrow P_{1} P_{2} \in \mathcal{S}_{\mathfrak{A}}^{m_{1}+m_{2}},
$$

and

$$
O P S_{1,0}^{m}(M) \subset \mathcal{S}_{\mathfrak{A}}^{m}
$$

In particular, for an operator $E: C^{\infty}(M) \rightarrow \mathcal{D}^{\prime}(M)$,

$$
E L \in \mathcal{S}_{\mathfrak{A}}^{m} \Rightarrow E \in \mathcal{S}_{\mathfrak{A}}^{m-2},
$$

since $L^{-1} \in O P S^{-2}(M)$. Consequently we see that, if $\mathfrak{A}_{b}$ is the set of $A_{j}$ over which we sum in (1.2),

$$
E A_{j} \in \mathcal{S}_{\mathfrak{A}}^{m} \text { for all } A_{j} \in \mathfrak{A}_{b} \Rightarrow E \in \mathcal{S}_{\mathfrak{A}}^{m-1} .
$$

Now suppose $B_{l} \in O P S^{0}(M)$ are such that

$$
B_{l} A_{j} \in \mathfrak{A} \text { for all } A_{j} \in \mathfrak{A}_{b} .
$$

Since

$$
\left(\operatorname{ad} B_{l} \cdot P\right) A_{j}=\operatorname{ad}\left(B_{l} A_{j}\right) \cdot P-B_{l}\left(\operatorname{ad} A_{j} \cdot P\right),
$$

we deduce that ad $B_{l} \cdot P \in \mathcal{S}_{\mathfrak{A}}^{m-1}$ if $P \in \mathcal{S}_{\mathfrak{A}}^{m}$. We hence deduce the following:

Proposition 1.7. If $(\operatorname{ad} C)^{J}=\operatorname{ad} C_{j_{l}} \cdots \operatorname{ad} C_{j_{1}}$, where $\mu$ of the $C_{j}$ 's are $B_{j}$ 's, satisfying (1.14), and the rest are $A_{j}$ 's, in $\mathfrak{A}$, then

$$
P \in \mathcal{S}_{\mathfrak{A}}^{m} \Rightarrow(\operatorname{ad} C)^{J} P \in \mathcal{S}_{\mathfrak{A}}^{m-\mu} .
$$

We note that this result applies when $\mathfrak{A}$ consists of all smooth vector fields on $M$ and the $B_{l}$ are multiplication operators, by smooth functions on $M$. In this case, Proposition 1.7 is one simple step in the well known result of Cordes (et al.) that $\mathcal{S}_{\mathfrak{A}}$ is precisely $O P S_{1,0}^{0}(M)$. 


\section{2. $U$-Smooth operators; A CHARACTERIZATION of $O P S_{1,0}^{0}(M)$}

Let $M$ be a compact smooth manifold, and $G$ a Lie group, with a strongly continuous unitary representation $U(g)$ on $L^{2}(M)$. Let $\left\{X_{j}: 1 \leq j \leq m\right\}$ be a basis of the Lie algebra $\mathfrak{g}$. Suppose $d U\left(X_{j}\right)=i A_{j} \in O P S^{1}(M)$. Also suppose that, if $\Delta$ is the Laplace operator on $G$, with a left invariant metric, then $d U(\Delta)=L$ is an elliptic operator in $O P S^{2}(M)$; one could take $L=-\sum A_{j}^{2}$.

Let $P \in \mathcal{L}\left(L^{2}(M)\right)$. We say $P$ is $U$-smooth if

$$
P(t)=U(t) P U(t)^{-1}
$$

is a $C^{\infty}$ function of $t \in G$, with values in $\mathcal{L}\left(L^{2}(M)\right)$. If $P$ is $U$-smooth, it follows that $P$ is $\mathfrak{A}$-smooth, with $\mathfrak{A}=\left\{A_{1}, \ldots, A_{m}\right\}$, i.e.,

$$
\operatorname{ad} A_{j_{l}} \cdots \operatorname{ad} A_{j_{1}} \cdot P: L^{2}(M) \rightarrow L^{2}(M)
$$

for all $j_{1}, \ldots, j_{l}$. We aim to show that, under condition (2.4) given below, we have boundedness of commutators when the $A_{\nu}$ are replaced by arbitrary operators in $O P S^{1}(M)$, so that the criterion discovered by [B], [C], and [D] will apply.

Identify a small neighborhood $\mathcal{O}$ of the identity element $e \in G$ with a neighborhood of 0 in $\mathfrak{g}$, via the exponential map. Suppose $\varphi \in \mathcal{E}^{\prime}(\mathcal{O})$ is a classical conormal distribution, with singularity at the origin, of the form $\varphi=\hat{f}$, with $f$ in the symbol space $S^{\mu}\left(\mathfrak{g}^{*}\right)$. Set

$$
f(A)=U(\hat{f})=\int \hat{f}(t) \kappa(t) U(t) d t
$$

where $\kappa(t) d t$ is Haar measure (in exponential coordinates). For $\mu \ll 0$, this is readily verified to be smoothing, via the following simple result.

Lemma 2.1. If $\varphi \in C_{0}^{2 k}(G)$, then $U(\varphi): H^{s}(M) \rightarrow H^{s+2 k}(M)$, for all $s \in$ $[-2 k, 0]$.

Proof. For $s=0$, this follows from the identity

$$
L^{k} U(\varphi)=U\left(\Delta^{k} \varphi\right)
$$

Then we have the result for $s=-2 k$ by duality, and the rest by interpolation.

A more precise analysis of (2.2) is provided by the following result, proved in Appendix B (pp. 163-165) of [T3], following material developed in [T1] and in Chapter 12 of [T2].

Lemma 2.2. If $f \in S^{\mu}\left(\mathfrak{g}^{*}\right)$, with $\hat{f}$ supported in $\mathcal{O}$, then $f(A) \in O P S^{\mu}(M)$, and the principal symbol of $f(A)$ is equal to $f\left(a_{1}, \ldots, a_{m}\right)$ on $T^{*} M \backslash 0$, where $a_{j}(x, \xi)$ is the principal symbol of $A_{j}$.

We now state our hypotheses on the action $U$. Consider the 'moment map'

$$
\Phi(x, \xi)=\left(a_{1}(x, \xi), \ldots, a_{m}(x, \xi)\right) ; \quad \Phi: T^{*} M \backslash 0 \rightarrow \mathbb{R}^{m} \backslash 0 .
$$

The hypothesis is:

$$
\Phi: T^{*} M \backslash 0 \rightarrow \mathbb{R}^{m} \backslash 0 \text { is an imbedding. }
$$

An equivalent statement is that for any $q_{\mu} \in S^{\mu}\left(T^{*} M \backslash 0\right)$, homogeneous of degree $\mu$, there exists a homogeneous $f_{\mu} \in S^{\mu}\left(\mathfrak{g}^{*}\right)$ such that

$$
q_{\mu}(x, \xi)=f_{\mu}\left(a_{1}(x, \xi), \ldots, a_{m}(x, \xi)\right) .
$$


In light of Lemma 2.2, we see that, if $Q \in O P S^{\mu}(M)$ has principal symbol $q_{\mu}$, then $Q=f_{\mu}(A), \bmod O P S^{\mu-1}(M)$. An inductive argument easily gives the following.

Proposition 2.3. Under hypothesis (2.4), given any $Q \in O P S^{\mu}(M)$, there exists $f \in S^{\mu}\left(\mathfrak{g}^{*}\right)$ such that $Q=f(A), \bmod O P S^{-\infty}(M)$, and such that $\operatorname{supp} \hat{f} \subset \mathcal{O}$.

We now examine the commutator of an operator $f(A)$, given $f \in S^{1}\left(\mathfrak{g}^{*}\right)$, with an operator $P \in \mathcal{L}\left(L^{2}(M)\right)$, assumed to be $U$-smooth. Using exponential coordinates, set

$$
P(t) \sim \sum_{\beta \geq 0} P_{\beta} t^{\beta}, \quad P_{\beta}=D^{\beta} P(0) / \beta !,
$$

where $P(t)$ is given by $(2.1)$. It easily follows that each $P_{\beta}$ is $U$-smooth, if $P$ is. Using

$$
U(t) P=P(t) U(t) \sim \sum_{\beta \geq 0} P_{\beta} t^{\beta} U(t),
$$

and plugging into (2.2), we get

$$
f(A) P \sim P f(A)+\sum_{\beta>0} P_{\beta} \int \hat{f}(t) t^{\beta} \kappa(t) U(t) d t \sim \operatorname{Pf}(A)+\sum_{\beta>0} P_{\beta} f_{\beta}(A) .
$$

Note that

$$
f \in S^{1}\left(\mathfrak{g}^{*}\right) \Rightarrow f_{\beta} \in S^{1-|\beta|}\left(\mathfrak{g}^{*}\right) \Rightarrow f_{\beta}(A) \in O P S^{1-|\beta|}(M) .
$$

Since it is clear that each element of $O P S_{1,0}^{0}(M)$ is $U$-smooth, and the set of $U$ smooth operators forms an algebra, we deduce the following:

Proposition 2.4. If $P$ is $U$-smooth, then for all $f \in S^{1}\left(\mathfrak{g}^{*}\right)$, with $\hat{f}$ supported in $\mathcal{O}$,

$$
[f(A), P]: L^{2}(M) \rightarrow L^{2}(M),
$$

and furthermore this commutator is $U$-smooth.

It follows that, for any $f_{\nu} \in S^{1}\left(\mathfrak{g}^{*}\right)$, with $\hat{f}_{\nu}$ supported in $\mathcal{O}$, the iterated commutators

$$
\text { ad } f_{N}(A) \cdots \text { ad } f_{1}(A) \cdot P
$$

are all bounded on $L^{2}(M)$, if $P$ is $U$-smooth. In view of Proposition 2.3, the criterion of [B], [C] applies: for any $Q_{\nu} \in O P S^{1}(M)$,

$$
\operatorname{ad} Q_{N} \cdots \operatorname{ad} Q_{1} \cdot P: L^{2}(M) \rightarrow L^{2}(M) .
$$

This gives our main conclusion:

Theorem 2.5. Under hypothesis (2.4), an operator $P \in \mathcal{L}\left(L^{2}(M)\right)$ is in $O P S_{1,0}^{0}(M)$ if and only if it is $U$-smooth.

We make some remarks on the hypothesis (2.4). Note that, for $(x, \xi) \in T^{*} M \backslash 0$,

$$
\begin{aligned}
D \Phi(x, \xi) \text { is injective } & \Leftrightarrow\left\{d a_{j}(x, \xi): 1 \leq j \leq m\right\} \text { spans } T_{(x, \xi)} T^{*} M \\
& \Leftrightarrow\left\{H_{a_{j}}: 1 \leq j \leq m\right\} \text { spans } T_{(x, \xi)} T^{*} M,
\end{aligned}
$$

where $H_{a_{j}}$ are Hamiltonian vector fields, generating an associated action $U^{\#}$ of $G$ on $T^{*} M \backslash 0$. This last condition holds provided

$$
G \text { acts transitively on } T^{*} M \backslash 0 \text {, via } U^{\#} \text {; }
$$


in fact when $T^{*} M \backslash 0$ is connected these conditions are equivalent. We also note that the moment map is invariantly defined as

$$
\Phi: T^{*} M \backslash 0 \rightarrow \mathfrak{g}^{*} \backslash 0 ;
$$

see [GS]. Furthermore, $\Phi$ intertwines the $U^{\#}$ on $T^{*} M \backslash 0$ with the $A d^{*}$ action of $G$ on $\mathfrak{g}^{*} \backslash 0$. The condition (2.9) implies that, under the image of $\Phi, T^{*} M \backslash 0$ covers a coadjoint orbit of $G$ in $\mathfrak{g}^{*} \backslash 0$. The hypothesis (2.4) would state that $\Phi$ maps $T^{*} M \backslash 0$ diffeomorphically onto such a coadjoint orbit.

An important example of this phenomenon is provided by the natural action of the conformal group $S O_{e}(n+1,1)$ on the sphere $S^{n}$,

$$
U(g) v(x)=J_{g}(x)^{1 / 2} v\left(g^{-1} \cdot x\right),
$$

where $J_{g}(x)$ is the usual Jacobian determinant. Thus Theorem 2.5 applies in this case. Thus we have proved the Theorem stated in the introduction.

We note that this $U$-action on $L^{2}\left(S^{n}\right)$ is the simplest sort of principal series representation of the Lie group $S O_{e}(n+1,1)$. Given a general irreducible unitary representation $U$ of a Lie group $G$ on a Hilbert space $H$, one can consider the set of operators $P$ on $H$ such that the family (2.1) is smooth from $G$ to $\mathcal{L}(H)$. As another sort of example, we mention the following result of [C]; see also [C2]. If $G$ is the Heisenberg group (of dimension $2 n+1$ ) and $U$ the standard representation on $L^{2}\left(\mathbb{R}^{n}\right)$, then an operator $P$ is $U$-smooth if and only if $P \in O P S_{0,0}^{0}\left(\mathbb{R}^{n}\right)$. It is of interest to consider families of $U$-smooth operators in other situations, but we will not pursue this here.

\section{REFERENCES}

[B] R. Beals, Characterization of pseudodifferential operators and applications, Duke Math. J. 44 (1977), 45-57; correction 46 (1979), 215. MR 55:8884; MR 80b:47062

[C] H. Cordes, On pseudodifferential operators and smoothness of special Lie group representations, Manuscripta Math. 28 (1979), 51-69. MR 80m:47047

[C2] H. Cordes, The technique of pseudodifferential operators, LMS \#202, Cambridge Univ. Press, 1995. MR 96b:35001

[D] J. Duneau, Fonctions d'un operateur elliptique sur une variete compacte, J. Math. Pures et Appl. 56 (1977), 367-391.

[GY] J. Goodman and D. Yang, Local solvability of nonlinear partial differential equations of real principal type, Preprint, 1987.

[GS] V. Guillemin and S. Sternberg, Symplectic techniques in physics, Cambridge Univ. Press, 1984. MR 86f:58054

[H] R. Hamilton, The inverse function theorem of Nash-Moser, Bull. AMS 7 (1982), 65-222. MR 83j:58014

[P] K. Payne, Smooth Frechet algebras and Lie groups of pseudodifferential operators, Comm. Pure Appl. Math. 44 (1991), 309-337. MR 92a:58140

[T1] M. Taylor, Fourier integral operators and harmonic analysis on compact manifolds, Proc. Symp. Pure Math. 35 (Part 2) (1979), 115-136. MR 81i:58042

[T2] M. Taylor, Pseudodifferential operators, Princeton Univ. Press, 1981. MR 82i:35172

[T3] M. Taylor, Noncommutative Microlocal Analysis, Part I, Memoirs AMS \#313, 1984. MR 86f:58156

Department of Mathematics, University of North Carolina, Chapel Hill, North CarOLINA 27599-3902

E-mail address: met@math.unc.edu 